\title{
L'infestation cercarienne chez Gibbula umbilicalis Da Costa
}

\author{
Par Lucie ARVY
}

\begin{abstract}
« Since experimental demonstration of trematode life histories is so difficult and slow, progress must be made... by the accumulated knowledge of these organisms, acquired bit by bit through the observation of different workers. \#
\end{abstract}

STUNKARD, 1932.

En 1906, Pelseneer signalait qu'il avait observé, une seule fois en dix ans, sur des milliers de Trochus cinerarius du Boulonnais qu'il avait examinés, des «sporocystes * allongés à nodosités et rétrécissements, jaunâtres et parfois même bruns-orangés, abritant de très nombreuses cercaires cystophores, absolument transparentes, incolores, à corps allongé et contractile, à ventouses subégales, la postérieure étant située, à peu près, à mi-corps ; Pelseneer avait pu suivre le développement de la cercaire et il a figuré des silhouettes, à divers stades de croissance.

Il a fallu attendre près d'un demi-siècle pour voir signaler à nouveau la même larve de Trématode ; en 1953, en effet, Gaillard semble avoir retrouvé Cercaria Vaullegeardi Pelseneer (1906) dans des «sporocystes blancs », chez un des 374 Gibbula umbilicalis qu'il avait récoltés aux îles Chausey. En outre, une cercaire très apparentée, sinon identique à $C$. Vaullegeardi a dernièrement été trouvée chez des Gibbula varia du grand aquarium du laboratoire Arago à Banyuls-sur-mer (Chabaud et Campana-Rouget, 1959) ; les «sporocystes d de cette cercaire cystophore comportant des zones, les unes blanches, les autres rougeâtres.

Contrastant avec la rareté des observations faites sur Cercaria Vaullegeardi Pelseneer (1906) de nombreux chercheurs ont vu des cercaires cotylicerques chez des gibbules ; l'ensemble des descriptions forme un tout extrêmement confus. La confusion a commencé à s'établir il y a plus d'un siècle avec la découverte de Lespès (1857). Travaillant à Arcachon, cet auteur a trouvé dans le testicule d'un gastropode qu'il qualifie * Trochus cynereus» une cercaire microcerque qu'il a malencontreusement dénommée Cercaria brachyura, dénomination appliquée antérieurement par Diesing (1850) à une cercaire parasite de Planorbis submarginata. Travaillant à Cette. Pagenstescher (1862) a dénommé Cercaria cotylura, une cercaire très apparentée à celle vue par Lespès (1857), trouvée chez un gastéropode prosobranche qu'il qualifie de Trochus cinereus * ; malheu-

* Il s'agissait probablement de Gibbula rarilineata (Michaud) ou de Gibbula divaricata (L.) (Dollfus, 1960). 
reusement ce troque n'existe pas en Méditerranée (Dollfus, 1913 ; Palombi, 1934). La question ne s'éclaircit pas avec Pelseneer (1906) ; travaillant à Wimereux, cet auteur

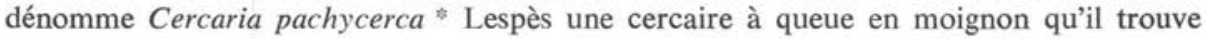
dans des sporocystes bruns-orangés, « marbrés », occupant la loge génitale de Trochus cinerarius; cependant que travaillant à Millport, Lebour (1912) dénomme Cercaria brachyura Lespès, une cercaire cotylicerque qu'elle trouve dans des sporocystes orangés, chez Gibbula (= Trochus) cinerea. Or, comme le fait remarquer Stunkard (1932) la cercaire décrite par Lebour (1912) est si différente de celles décrites par Lespès (1857) et par Pelseneer (1906), chez le même hôte, Gibbula cinerea, qu'elle ne peut leur être homologuée.

En 1932, Stunkard dénomme Cercaria brachyura Lespès, provis., des cercaires cotylicerques qu'il trouve, éparpillées dans un récipient ayant contenu cent Gibbula umbilicalis (= Gibbus umbilicatus) récoltés à Roscoff. Or, comme le fait remarquer Palombi (1934) l'espèce décrite par Stunkard diffère de Cercaria brachyura Lespès (1857, non seulement par sa taille — qui semble être inférieure — mais encore par la forme du corps, le diamètre des ventouses et l'appareil excréteur ; de sorte que Palombi propose la transformation de cette $C$. brachyura provis. en Cercaria Stunkardi.

En 1953, Gaillard homologue une cercaire cotylicerque à sporocystes jaunes, parasite de la gonade des Gibbula umbilicalis et $G$. cinerea, à la fois à Cercaria cotylura Pagenstescher (1862) et à Cercaria pachycerca Lespès (1857). Dollfus enfin (1960), décrit sept cercaires cotylicerques (A, B, C, D, E, H et I) parasites de Gibbula cineraria $\mathrm{L}$ à Roscoff et une autre, dite $\mathrm{G}$, parasite de Gibbula umbilicalis (Da Costa) à SaintVaast-la-Hougue (Manche) ; cependant aucune de ces cercaires n'évolue dans des sporocystes jaunes et seules les Cercaria cotylicerca $\mathrm{G}$ et $\mathrm{H}$ sont inféodées à la gonade. Malheureusement Dollfus (1960) n'établit aucune correspondance entre ses cercaires cotylicerques et celles qui ont été décrites antérieurement chez les Gibbules.

Dès 1932, Stunkard estimait qu'il ne pouvait en toute certitude rapporter les cerzaires cotylicerques qu'il observait à Roscoff à quelqu'une des cercaires connues ; je ne puis avoir la prétention de faire mieux que Stunkard. Cependant si l'on se fie à la coloration des sporocystes la cercaire cotylicerque vue dans la gonade de Trochus cinerarius par Pelseneer (1906) et dénommée $C$. pachycerca Lespès est identique à celle vue par Lebour (1912) dans la glande digestive de Gibbu'a cinerea et dénommée $C$. brachyura Lespès, comme elle est identique à la $C$. pachycerca de la gonade des Gibbula cineraria et G. umbilicalis de Gaillard (1953), l'appellation correcte de cette cercaire devant être soit $C$. brachyura Lespès (1857) soit $C$. pachycerca Diesing (1858) ; la place de Cercaria Stunkardi (Palombi, 1934) restant à déterminer, puisque les sporocystes de cette espèce sont inconnus. Quoiqu'il en soit, mon intention n'est pas d'ajouter à la confusion des Gibbules et de leurs parasites ; je veux seulement rapporter quelques observations faites

* Il n'existe aucune parenté entre cette cercaire et la Cercaria pachycerca [découverte libre, dans [e plancton, à Saint-Vaast-la-Hougue (Manche)], en 1863, par A.-R.-E. Claparède [et revue par Leuckart - si on en croit Carus (1884) et Parona (1894) - dans la cavité gastro-ventriculaire de Cydippe sp. = Pleurobranchia, à Nice], cercaire à énorme queue plate, annelée, aussi large et cinq fois plus longue que le corps. 


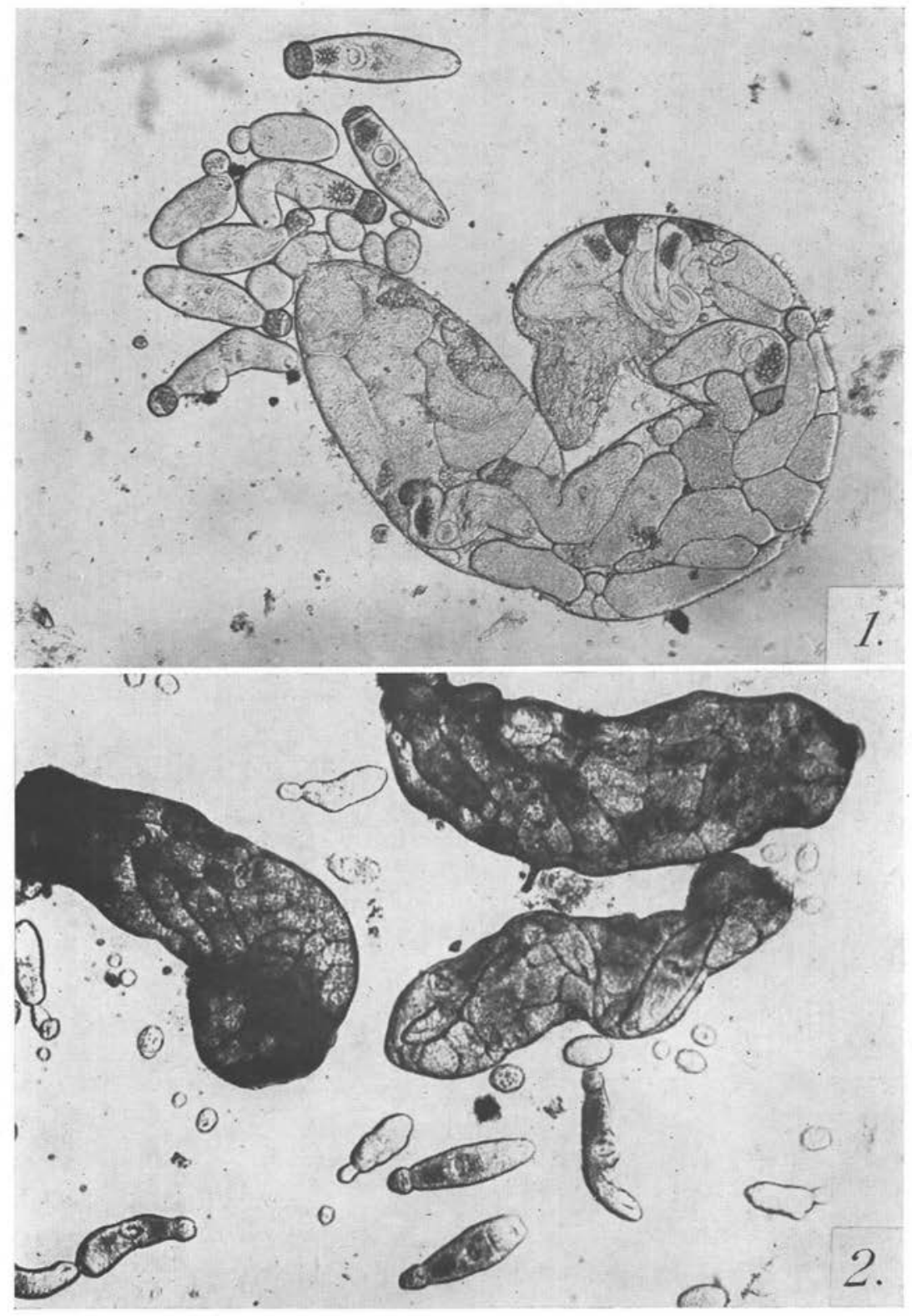

Fig. 1 et 2. - Sporocystes de cercaires cotylicerques parasites de la gonade de Cercaria umbilicalis Da Costa. Fig. 1: sporocyste blanc et ses cercaires. Fig. 2: sporocyste orangé et ses cercaires. Photographies du matériel vivant, faites au même grossissement, par le $\mathrm{D}^{r}$ R. Lami 
durant trois courts séjours dans les laboratoires maritimes de Roscoff (Finistère) et de Dinard (Ille-et-Vilaine).

\section{Recherches personnelles.}

Contrairement à l'assertion de Pelseneer (1906) suivant laquelle les Mollusques intercotidaux: Patella vulgata L, Littorina littorea L, Trochus umbilicalis Da Costa, etc... ne sont jamais parasités par des cercaires, mes dissections de Gibbu!a umbilicalis Da Costa, réparties sur deux ans, m'ont toujours permis de trouver une infestation par trois types de cercaires. Les parthénopes de ces types sont représentés dans les figures 1 , 2, 3. Les sporocystes (fig. 1 et 2 ) occupent la loge génitale provoquant le plus souvent la castration de la Gibbule ; ils contiennent des cercaires cotylicerques. Les rédies (fig 3) sont cantonnées entre les lobules de la glande médio-intestinale ; elles contiennent des cercaires cystophores.

\section{$1^{\circ}$ Les cercaires cotylicerques' :}

Les photographies 1 et 2 prises sur le vivant * sont plus précises que de longs discours. Il n'est pas douteux que les Gibbula umbilicalis Da Costa hébergent deux types de cercaires cotylicerques, très proches l'un de l'autre, mais parfaitement distinguables lorsqu'on s'adresse aux cercaires et totalement différents quand on s'adresse aux sporocystes, les uns blancs, les autres orangés.

Les sporocystes blancs (fig. 1) ont une paroi mince, très transparente et souple ; ils contiennent de nombreuses cercaires à queue sombre, dont la ventouse ventrale est en arrière du milieu du corps.

Les sporocystes orangés (fig. 2) sont trapus à paroi épaisse, raide, presque opaque ; ils contiennent des cercaires à queue claire, à ventouse ventra'e située au milieu du corps.

Les deux types de parasites sont évidents dès le décoquillage du gastéropode ; la gonade de ce dernier, vert-jade chez les $q$, blanche laiteuse plus ou moins rosée chez Dinard.

* Grâce à l'extrême obligeance du $D^{r}$ R. Lami, sous-directeur du Laboratoire maritime de

FIG. 3. - Rédie de Cercaria Vaullegeardi Pelseneer, dont ia partie postérieure (à gauche) est très contractée, longueur réelle: $2 \mathrm{~mm} 50$ (Photo $\mathrm{R}$. Lami; rédie vivante).

FIG. 4. - Coupe de tortillon de Gibbula umbilicalis Da Costa après coloration par l'hémalunpicro-indigocarmin. La gonade (à droite) est normale, de même que la zone gauche de la glande médio-intestinale; le reste de la glande est farci de cercaires cystophores

Fig. 5. - Coupe de glande médio-intestinale de G. umbilicalis un peu dissociée pour montrer la minceur de la rédie; par place, elle n'excède pas la largeur de deux ou trois cercaires (= environ 60 à 90 ఓ)

Fig. 6. - Photographie en contraste de phase de Cercaria Vaullegeardi Pelseneer, vivante, par le $D^{r}$ R. Lami

FIG. 7. en bas, à gauche. - Coupe de la glande médio-intestinale chez G. umbilicalis, dans une zone riche en rédies, colorée par l'acide périodique Schiff; les sections des appendices contenus dans le cyste apparaissent punctiformes 

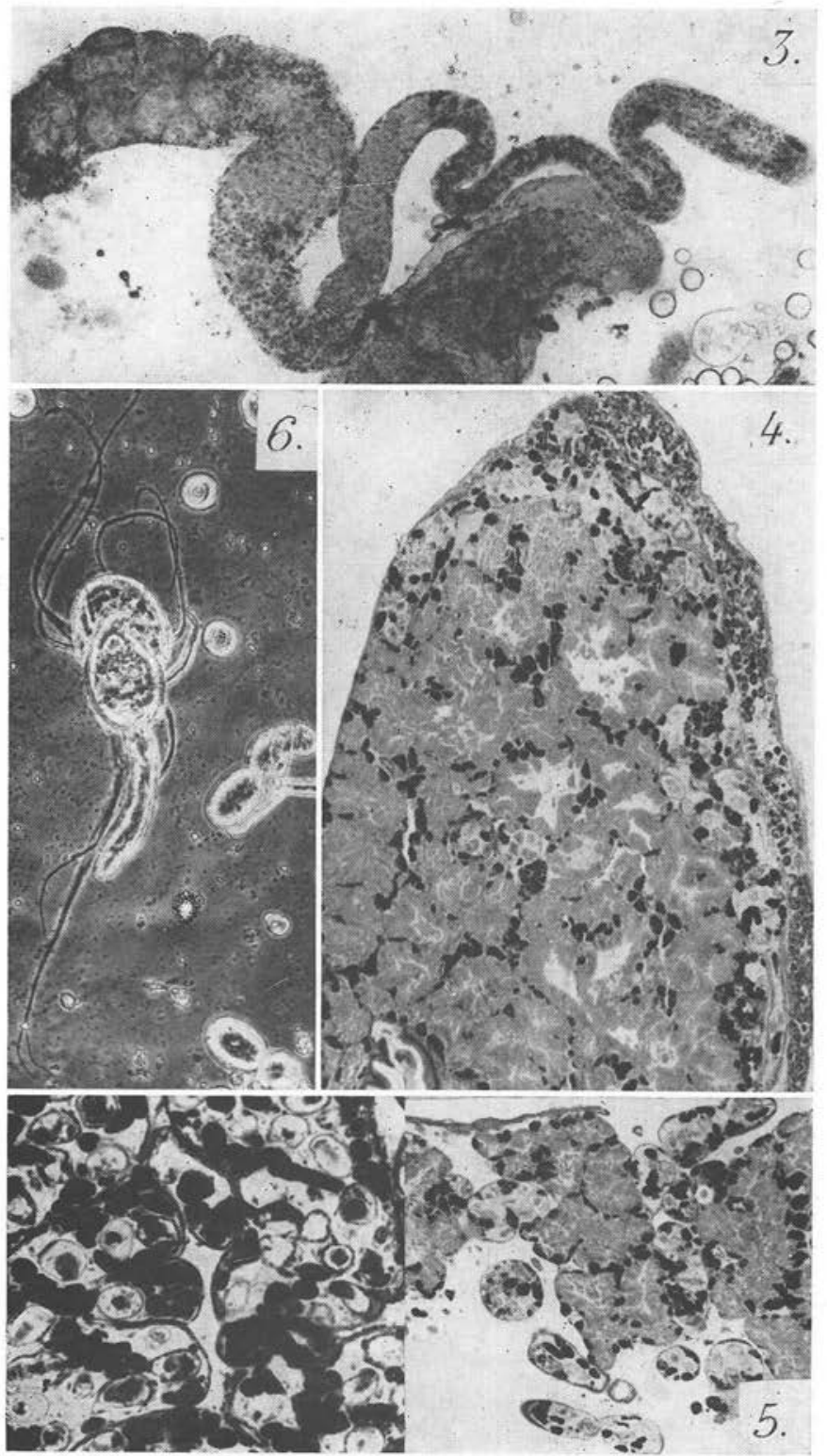
les $\delta$, est remplacée soit par des plaques d'un blanc gélatineux avec les cercaires du premier type, soit par des masses d'un bel orangé avec les cercaires du deuxième type.

Ces deux types d'infestation par des cercaires cotylicerques sont également fréquents chez les Gibbula umbilicalis de Roscoff et chez celles de Dinard. L'infestation par les cercaires à sporocystes blancs est toujours plus fréquente (environ $6 \%$ ) que celle par les cercaires à sporocystes orangés (environ $1 \%$ ) ; à deux reprises j'ai trouvé une infestation mixte, la dissection permettant d'isoler les deux types de sporocystes.

\section{$2^{\circ}$ Les cercaires cystophores :}

Une dissection soigneuse permet toujours (environ $2 \%$ ) de retrouver, chez Gibbula umbilicalis de Roscoff, la cercaire cystophore vue à Wimereux chez Gibbula cineraria (Pelseneer, 1906) et aux îles Chausey chez Gibbula umbilicalis (Gaillard, 1953) ; cependant la dissection reste infructueuse chez les Gibbula umbilicalis de Dinard (baie du Prieuré).

L'infestation des Gibbula umbilicalis Da Costa par Cercaria Vaullegeardi Pelseneer n'est pas, comme l'infestation par les cercaires cotylicerques, évidente dès le décoquillage du gastéropode. En effet les rédies sont tubuliformes, voir filiformes (fig. 5) disposées entre les lobules de la glande médio-intestinale (fig. 4 ê̂ 5); il faut effondrer cette dernière pour isoler entièrement les rédies en bon état (fig. 3) ; elles apparaissent alors translucides, molles, très extensibles et fragiles. Elles contiennent de nombreuses cercaires cystophores, relativement petites, très caractéristiques, grâce à leurs nombreux appendices (fig. 6) et bien différentes de Cercaria Prenanti (Arvy, 1949-57, et Ching, 1960), parasites des Dentales, de Cercaria Dollfusi (Arvy, 1957), parasite de Philine aperta L. et de Cercaria Tregouboffi, parasite de Columbella rustica L. (Arvy, 1952). La chambre caudale semble pourvue de six longs filaments disposés par paires; elle abrite en outre deux appendices plus trapus, dont un rubanné, extrêmement long. Des coupes histologiques orientées convenablement, permettent, avec certaines colorations, de retrouver (fig. 7) les appendices filiformes sous forme de points dans les chambres caudales. Les cercaires et leurs appendices sont d'un fragilité exquise, de sorte que l'observateur se trouve devant une pénible alternative : ou bien les cercaires sont parfaitement vivantes et les contractions simultanées de la cercaire proprement dite, de sa chambre caudale et de ses appendices sont gênants, ou bien les cercaires sont mourantes et faciles à observer, mais elles apparaissent boudinées avec des appendices rompus, rétractés ou gonflés (fig. 8). Dans les cas les plus favorables on peut assister au refuge des cercaires en bon état dans leur chambre caudale (fig. 8).

L'examen de plus d'un millier de G. umbilicalis Da Costa de la baie du Prieuré à Dinard ne m'a pas permis de retrouver Cercaria Vaullegeardi Pelseneer. Cependant, à deux ans d'intervalle (octobre-novembre 1960 et 1962) j'ai retrouvé sans difficulté et avec la même fréquence d'infestation les cercaires cotylicerques à sporocystes blancs (fig. 1) et à sporocystes orangés (fig. 2) chez les G. umbilicalis de Dinard.

Un bref séjour à Roscoff en mai 1961 m'ayant permis de trouver sans difficulté les deux types de cercaires cotylicerques et Cercaria Vaullegeardi il restait à savoir si la différence d'infestation observée entre les Gibbules de Dinard et celles de Roscoff était 


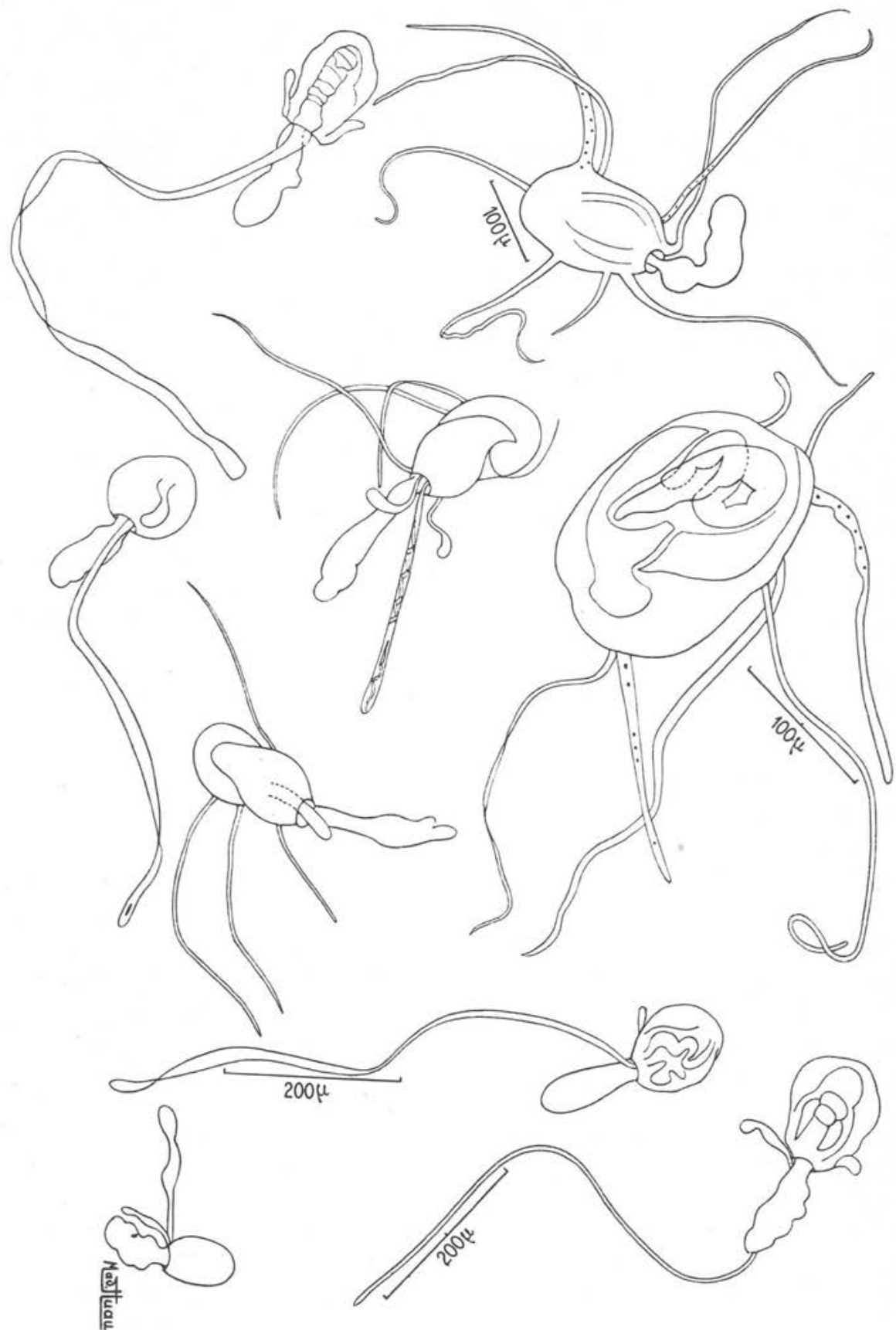

Fig. 8. - Dessins à la chambre claire de Cercaria Vaullegeardi vivante, dont une encystée. Ann. de Parasitologie humaine et comparée (Paris), t. 38, 1963, $\mathrm{n}^{\circ} 5$ 
d'otdre saisonnier ou d'ordre géographique. Un lot important de Gibbula umbilicalis Da Costa reçu de Roscoff le 10 novembre 1962 m'a permis de retrouver la cercaire cystophore et les deux cercaires cotylicerques vues à Roscoff en mai 1961, au moment même ou Cercaria Vaullegeardi restait introuvable à Dinard.

Il semble donc que certains facteurs d'infestation existent à Roscoff qui manquent à Dinard.

\section{Conclusion.}

Bien que souvent exondées les Gibbula umbilicalis Da Costa sont assez fréquemment parasitées par trois types de cercaires. Deux sont cotylicerques et s'attaquent à la gonade ; l'une est à sporocystes b'ancs, l'autre à sporocystes jaunes. Le troisième type est cystophore et rappelle en tous points la cercaire dédiée à Vaullegeard par Pelseneer en 1906 ; les rédies de cette cercaire s'attaquent à la glande médio-intestinale et ne lèsent pas directement la gonade des Gibbules.

\section{Bibliographie}

ARvy L., 1949. - Sur la castration parasitaire chez Dentalium entale Deshayes, C.R. Acad. 228,780 .

- 1949. - Présentation de documents relatifs à l'ovogénèse du dentale et à deux parasites de ce Scaphopode: Cercaria Prenanti n. sp. et Haplosporidium dentali n. sp. Bull. Soc. Zool. Fr., 74, 292.

- 1951. - Contribution à l'étude de Cercaria Dollfusi, cercaire cystophore parasite de Philine aperta L (Opisthobranche Céphalaspide). Ibid., 76, 339-48, 21 fig.

- 1952. - Présentation de documents relatifs à trois cercaires parasites de Columbella rustica L (Gastéropode prosobranche). Ibid., 77, 217-20.

- 1952. -- Contribution à l'étude des Trématodes parasites de Columhella rustica (Gastéropode prosobranche). Ann. parasitol., 27, 485-98, 16 fig.

— 1957. - Contribution à l'étude du parasitisme chez le Dentale. Arch. für. Zellf., 45, 444-63.

Chabaud A.-G. et Biguet J., 1954. - Sur le mécanisme d'infestation des Copépodes par les cercaires de trématodes hémiuroïdes. C.R. Acad. Sci., 239, 1087.

— 1955. — Etude d’un Trématode hémiuroïde à métacercaire progénétique. I. - Développement chez le Mollusque. II. - Infestation du Copépode. III. - Développement chez le Copépode. Ann. Parasitol., 29, 527-45.

Chabaud A.-G. et Campana-Rouget, 1959. - Notes sur le trématode hémiuroïde Sterrhurus fusiformis Lühe 1901 et sur sa cercaire (Cercaria Vaullegeardi Pelseneer, 1906), Vie et Mil'eu, 10, 168-75.

Ching H. L., 1960. - Studies on three hemiuroid cercariae from Friday harbor, Washington. J. Parasitol, 46, 663-70.

DiesING K. M., 1858. - Berichtigungen und Zusätze zur Revision der Cercarien. Sitz Akad. Wis. Wien Math. natur-wis, 31, 239-90. 
Dollfus R. Ph., 1960. - Caractéristiques spécifiques des cercaires cotylicerques. Ann. Parasitol., 35, 29-81, 39 fig.

- 1950. - Notes et distribution géographique des cercaires cystophores. Ann. Parasitol., 25, 276-96.

Gaillard J. M., 1953, - Sur quelques points d'anatomie et de biologie de Gibbula umbilicalis Da Costa (Mollusque prosobranche). Bull. Lab. Mar. Dinard, 39, 1-21.

Lebour M., 1912. - A review of the British marine Cercariae. Parasitology, 4, 416-56, 5 pl.

Pagenstescher H. A., 1862. - Untersuchungen über einige Seethiere aus Cette. Z. wis. Zool., $12,265-311,5 \mathrm{pl}$.

Palombi A., 1934. - Gli stadi larvali dei Trematodi del golfo di Napoli. Contributo allo studio della morfologia, biologia e sistematica delle cercarie marine. Publ. Staz. Zool. Napoli, 14, 51-94.

- 1938. - Gli stadi larvali dei Trematodi del golfo di Napoli. Contributo allo studio della morfologia, biologia et sistematica della cercarie marine (Il gruppo delle cercaria cotylocerche). Revist. Parassitol., 2, 189-206.

LESPÈs Ch., 1857. - Observations sur quelques cercaires parasites de Mollusques marins. Ann. Sci. nat., 7, 113-7, 1 pl. ..

Pelseneer P., 1906. - Trématodes parasites de Mollusques marins. Bull. Biol. Fr. Belg., 40, 161-86, 5 pl., 161-86.

StunKaRd H. W., 1932. - Some larval Trematodes from the coast in the region of Roscoff, Finistère. Parasitology, 24, 321-43.

(Laboratoire de Physiologie du C.N.R.Z. et Laboratoire maritime de Dinard). 